\title{
NEW M, L, AND T DWARF COMPANIONS TO NEARBY STARS FROM THE WIDE-FIELD INFRARED SURVEY EXPLORER*
}

\author{
Kevin L. Luhman ${ }^{1,2}$, Nicholas P. Loutrel ${ }^{1}$, Nicholas S. McCurdy ${ }^{1}$, Gregory N. Mace ${ }^{3}$, Nicole D. Melso ${ }^{1}$, \\ Kimberly M. StaR ${ }^{1}$, Michael D. Young ${ }^{4}$, Ryan C. Terrien ${ }^{1}$, Ian S. McLean ${ }^{3}$, \\ J. DAVy KirKPatrick ${ }^{5}$, AND Katherine L. RHODE ${ }^{4}$ \\ ${ }^{1}$ Department of Astronomy and Astrophysics, The Pennsylvania State University, University Park, PA 16802, USA; kluhman@astro.psu.edu \\ ${ }^{2}$ Center for Exoplanets and Habitable Worlds, The Pennsylvania State University, University Park, PA 16802, USA \\ ${ }^{3}$ UCLA Division of Astronomy and Astrophysics, Los Angeles, CA 90095, USA \\ ${ }^{4}$ Department of Astronomy, Indiana University, Swain West 319, 727 East Third Street, Bloomington, IN 47405, USA \\ ${ }^{5}$ Infrared Processing and Analysis Center, MS 100-22, California Institute of Technology, Pasadena, CA 91125, USA \\ Received 2012 August 24; accepted 2012 October 15; published 2012 November 16
}

\begin{abstract}
We present 11 candidate late-type companions to nearby stars identified with data from the Wide-field Infrared Survey Explorer (WISE) and the Two Micron All Sky Survey (2MASS). Eight of the candidates are likely to be companions based on their common proper motions with the primaries. The remaining three objects are rejected as companions, one of which is a free-floating T7 dwarf. Spectral types are available for five of the companions, which consist of $\mathrm{M} 2 \mathrm{~V}, \mathrm{M} 8.5 \mathrm{~V}, \mathrm{~L} 5, \mathrm{~T} 8$, and $\mathrm{T} 8$. Based on their photometry, the unclassified companions are probably two mid-M dwarfs and one late-M/early-L dwarf. One of the T8 companions, WISE J142320.84+011638.0, has already been reported by Pinfield and coworkers. The other T8 companion, ULAS J095047.28+011734.3, was discovered by Burningham and coworkers through the United Kingdom Infrared Telescope Infrared Deep Sky Survey, but its companionship has not been previously recognized in the literature. The L5 companion, 2MASS J17430860+8526594, is a new member of a class of L dwarfs that exhibit unusually blue near-IR colors. Among the possible mechanisms that have been previously proposed for the peculiar colors of these L dwarfs, low metallicity does not appear to be a viable explanation for 2MASS J17430860+8526594 since our spectrum of the primary suggests that its metallicity is not significantly subsolar.
\end{abstract}

Key words: binaries: visual - brown dwarfs - infrared: stars - proper motions - stars: low-mass

Online-only material: color figures

\section{INTRODUCTION}

Among the hundreds of known $\mathrm{L}$ and $\mathrm{T}$ dwarfs in the solar neighborhood, the few dozen that reside in multiple systems with stars have the most accurately characterized physical properties because their ages and metallicities are relatively well constrained via their primaries. As a result, late-type companions to nearby stars have been highly sought after for testing models of the atmospheres and interiors of low-mass stars and brown dwarfs (Baraffe et al. 2003; Allard et al. 2011; Burrows et al. 2011; Saumon et al. 2012). Candidate companions have been initially identified through their colors and/or proper motions, which are measured through observations that target nearby stars as well as wide-field surveys like the Two Micron All Sky Survey (2MASS; Skrutskie et al. 2006), the Sloan Digital Sky Survey (SDSS; York et al. 2000), the SuperCOSMOS Sky Survey (SSS; Hambly et al. 2001), and the United Kingdom Infrared Telescope Infrared Deep Sky Survey (UKIDSS; Lawrence et al. 2007). More specifically, companion surveys have utilized near-infrared (near-IR) colors from targeted imaging (Becklin \& Zuckerman 1988) and 2MASS (Gizis et al. 2001), proper motions from seeing-limited (Mugrauer et al. 2006) and adaptive optics images (Nakajima et al. 1995; Potter et al. 2002; Metchev \& Hillenbrand 2006; Thalmann et al.

\footnotetext{
* Based on data from the Wide-field Infrared Survey Explorer, 2MASS, the W.M. Keck Observatory, the NASA Infrared Telescope Facility, the Hobby-Eberly Telescope, the WIYN Observatory at Kitt Peak National Observatory, the Spitzer Space Telescope, the Canada-France-Hawaii Telescope, and the European Southern Observatory New Technology Telescope.
}

2009), narrowband colors obtained with adaptive optics (Biller et al. 2006), and mid-IR colors (Luhman et al. 2007) and proper motions (Luhman et al. 2011) from the Spitzer Space Telescope (Werner et al. 2004). Additional companions have been found during surveys for free-floating $\mathrm{L}$ and $\mathrm{T}$ dwarfs, and later recognized as comoving with nearby stars (Faherty et al. 2010). Those surveys identified L/T candidates based on 2MASS colors (Burgasser et al. 2000; Kirkpatrick et al. 2001; Burgasser et al. 2005) and proper motions (Wilson et al. 2001), near-IR colors from UKIDSS (Burningham et al. 2009; Goldman et al. 2010; Day-Jones et al. 2011), optical colors from SDSS (Zhang et al. 2010), proper motions from UKIDSS and SDSS (Scholz 2010), and proper motions from SSS (Scholz et al. 2003).

The Wide-field Infrared Survey Explorer (WISE; Wright et al. 2010) and Pan-STARRS1 (PS1; Kaiser et al. 2002) are two of the newest resources for uncovering late-type companions in the solar neighborhood. Because WISE imaged the entire sky at mid-IR wavelengths, it is the best available tool for a widefield census of the coolest nearby brown dwarfs (Kirkpatrick et al. 2011; Cushing et al. 2011). PS1 does not reach the temperature limits of WISE since it operates at optical and near-IR wavelengths, but its combination of area, depth, spatial resolution, and temporal sampling greatly enhances the potential for proper motion surveys (Deacon et al. 2011; Liu et al. 2011). Late-type companions already have been discovered using colors from UKIDSS and WISE (Pinfield et al. 2012) as well as proper motions from 2MASS and WISE (Loutrel et al. 2011; Mužić et al. 2012) and 2MASS and PS1 (Deacon et al. 2012a, 2012b). In this paper, we present the latest discoveries 
Table 1

Spectral Types, Distances, and Astrometry for Candidate Binary Systems

\begin{tabular}{|c|c|c|c|c|c|c|c|c|c|}
\hline Primary & $\begin{array}{l}\text { Spectral } \\
\text { Type }\end{array}$ & $\begin{array}{l}\text { Distance } \\
\text { (pc) }\end{array}$ & $\begin{array}{c}\mu_{\alpha}, \mu_{\delta}^{\mathrm{a}} \\
\left(\mathrm{mas}_{\mathrm{yr}}{ }^{-1}\right)\end{array}$ & Ref & $\begin{array}{l}\text { Candidate } \\
\text { Companion }\end{array}$ & $\begin{array}{c}\text { Separation } \\
(\operatorname{arcsec} / \mathrm{AU})\end{array}$ & $\begin{array}{l}\text { Spectral } \\
\text { Type }\end{array}$ & $\begin{array}{c}\mu_{\alpha}, \mu_{\delta}^{\mathrm{a}, \mathrm{b}} \\
\left(\mathrm{mas}_{\mathrm{yr}}{ }^{-1}\right)\end{array}$ & $\begin{array}{c}\text { Likely } \\
\text { Binary? }\end{array}$ \\
\hline LPM J0318+0827S & $13 \mathrm{~V}$ & 2 & $(-234,-167) \pm 8$ & 2,3 & 2MASS J031 & $75 /$ & & $(-252,-1$ & yes \\
\hline LHS 1643 & mid-M? & 22 & $(+260,-573) \pm 8$ & $4,2,3$ & WISE J041328.73+821854.7 & $215 / 4700$ & & $(<300,<300)$ & no \\
\hline LHS 6176 & M3.5V & 27 & $(+241,-359) \pm 13$ & 2 & ULAS J095047.28+011734.3 & $52 / 1400$ & $\mathrm{~T} 8^{\mathrm{c}}$ & $(+245,-380) \pm 13$ & yes \\
\hline HD 91324 & F9V & & $(-419.3,+209.2) \pm 0.2$ & 5,6 & 2MASS J10313234-5338010 & $309 / 6700$ & $\ldots$ & $(-400,+211) \pm 23$ & yes \\
\hline LHS 2907 & G1V & 17.2 & $(+223.8,-477.4) \pm 0.4$ & 7,6 & WISE J142320.84+011638.0 & $156 / 2680^{\mathrm{d}}$ & $\mathrm{T} 8 \mathrm{p}^{\mathrm{e}}$ & $(+280,-420) \pm 60$ & yes \\
\hline 2MASS J14 & M7V & 27 & $(-314,-19) \pm 15$ & 8,2 & 2MASS J14351087-2333025 & $247 / 6700$ & M8V & $(-310,+54) \pm 15$ & no \\
\hline HD 147449 & F0V & 27.3 & $(-158.4,+49.6) \pm 0.4$ & 9,6 & 2MASS J16220644+0 & $43 / 1200$ & $\mathrm{M} 2 \mathrm{~V}$ & $(-155$, & yes \\
\hline G259-20 & $\mathrm{M} 2.5 \mathrm{~V}$ & 22 & $(-104.1,-269.5) \pm 2.7$ & 2,10 & 2MASS J17430860+8526594 & $30 / 650$ & L5 & $(-94,-271) \pm 25$ & yes \\
\hline HD 166620 & $\mathrm{~K} 2 \mathrm{~V}$ & 11.0 & $(-316.4,-468.5) \pm 0.3$ & 7,6 & WISE J180901.07+383805.4 & $769 / 8460$ & $\mathrm{~T} 7$ & $(-555,-48) \pm 60$ & no \\
\hline LHS 3421 & $\mathrm{M} 2.5 \mathrm{~V}$ & 25.9 & $(-246,-769) \pm 4$ & 11,6 & 2MASS J18525777-5708141 & $58 / 1500$ & $\ldots$ & $(-242,-743) \pm 35$ & yes \\
\hline LSPM J2010+0632 & M3.5V & 15 & $(+43,-195) \pm 8$ & 12,3 & 2MASS J20103539+0634367 & $143 / 2100$ & M8.5V & $(+51,-204) \pm 20$ & yes \\
\hline
\end{tabular}

Notes.

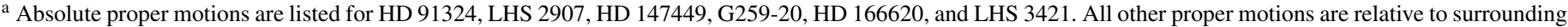
background stars.

b This work.

${ }^{c}$ B. Burningham 2012, in preparation.

d Pinfield et al. (2012) reported a separation of $153^{\prime \prime}$, which appears to be based on coordinates for the primary and secondary that are from different epochs.

e Pinfield et al. (2012).

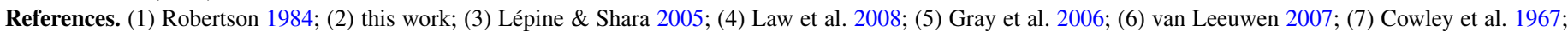
(8) Cruz et al. 2007; (9) Slettebak 1955; (10) Høg et al. 2000; (11) Hawley et al. 1996; (12) Shkolnik et al. 2010.

from our ongoing survey for late-type companions with 2MASS and WISE.

\section{SEARCH METHODS}

The primary sources of data for our survey for companions are the 2MASS Point Source Catalog and the WISE All-Sky Source Catalog. The images from 2MASS were collected between 1997 and 2001 in broadband filters centered at $1.25,1.65$, and $2.16 \mu \mathrm{m}$ ( $J, H, K_{s}$; Skrutskie et al. 2006). The WISE images were obtained during 2010 through filters at 3.4, 4.6, 12, and $22 \mu \mathrm{m}(W 1-W 4$, Wright et al. 2010).

Our companion survey consists of two complementary strategies. In the first one, we are searching for proper motion companions to stars and brown dwarfs within $\sim 30$ pc (e.g., Subasavage et al. 2005; Lépine 2005, 2008; Faherty et al. 2009) using astrometry from 2MASS and WISE. We discovered an L dwarf companion with this method shortly after WISE data became publicly available (Loutrel et al. 2011). Details of the data analysis are described in that study.

In our second survey strategy, we are searching for WISE sources that lack 2MASS counterparts and that exhibit WISE colors and magnitudes that are consistent with those expected for cool companions to nearby stars (Kirkpatrick et al. 2011, 2012). The photometric selection criteria consist of the following: $\sigma(W 2)<0.1, W 2-W 3<2.5$ if $\sigma(W 3)<0.25, W 1-W 2>$ $1(\gtrsim \mathrm{T} 1)$, and $2 \times W 2-W 1<\mathrm{DM}+11.5$, where $\mathrm{DM}$ is the distance modulus of the primary, which is computed from the parallax when available and the photometric distance otherwise. In addition to WISE sources that have no 2MASS counterparts within $3^{\prime \prime}$, we include WISE sources that are detected by 2MASS but that have uncertain photometry ( $>0.1 \mathrm{mag}$ ) in all $2 \mathrm{MASS}$ bands since they are excluded from the 2MASS/WISE proper motion search. For each candidate identified in this manner within a projected separation of 10,000 AU from the primary, we verify that it is unaffected by artifacts or blends in the WISE images and is not detected in optical images from the Digitized Sky Survey (DSS). We also attempt to measure the proper motion and further constrain the colors of each candidate by checking for detections in images from widefield surveys (e.g., UKIDSS) and the public data archives of observatories. If a candidate is sufficiently promising, we pursue follow-up imaging or spectroscopy to verify its cool nature and its companionship. For these follow-up observations, we give higher priority to candidates with smaller separations from the primary stars since they are more likely to be bona fide companions.

In the next section, we describe our new sample of 11 candidate companions to nearby stars. Table 1 lists the spectral types, proper motions, and distances of the primaries and spectral types, proper motions, and separations of the candidates. The 2MASS and WISE photometric data for the candidates are given in Table 2. Although we are primarily interested in finding $\mathrm{L}$ and $\mathrm{T}$ dwarf companions, we note the new $\mathrm{M}$ dwarf companions that have been uncovered as well. All candidates except the earliest one are plotted in color-magnitude and color-color diagrams in Figure 1, and those that are confirmed as companions based on their proper motions (see Table 1) are shown in a diagram of absolute magnitude versus spectral type in Figure 2. For reference, these diagrams also include samples of known M, $\mathrm{L}$, and $\mathrm{T}$ dwarfs. The $\mathrm{M}$ dwarfs are from the compilation of stars within 8 pc by Kirkpatrick et al. (2012) and the L and T dwarfs are from the compilations by Leggett et al. (2010), Kirkpatrick et al. (2011), and http://DwarfArchives.org. Because the $J$-band data for our candidate $\mathrm{T}$ dwarfs have been measured with the Mauna Kea Observatories (MKO) system, we adopt $J$-band data from that system for the known T dwarfs as well. For the known $\mathrm{M}$ and L dwarfs, we adopt $J, H$, and $K_{s}$ from 2 MASS.

\section{CANDIDATE COMPANIONS}

\subsection{MASS $03184214+0828002$}

Through our 2MASS/WISE proper motion analysis, we identified 2MASS J03184214+0828002 (hereafter 2M 0318+0828) as a possible companion to the 6" binary system LSPM J0318+0827S/N (Lépine \& Shara 2005). The brighter component of the binary, LSPM J0318+0827S, has a spectral type 

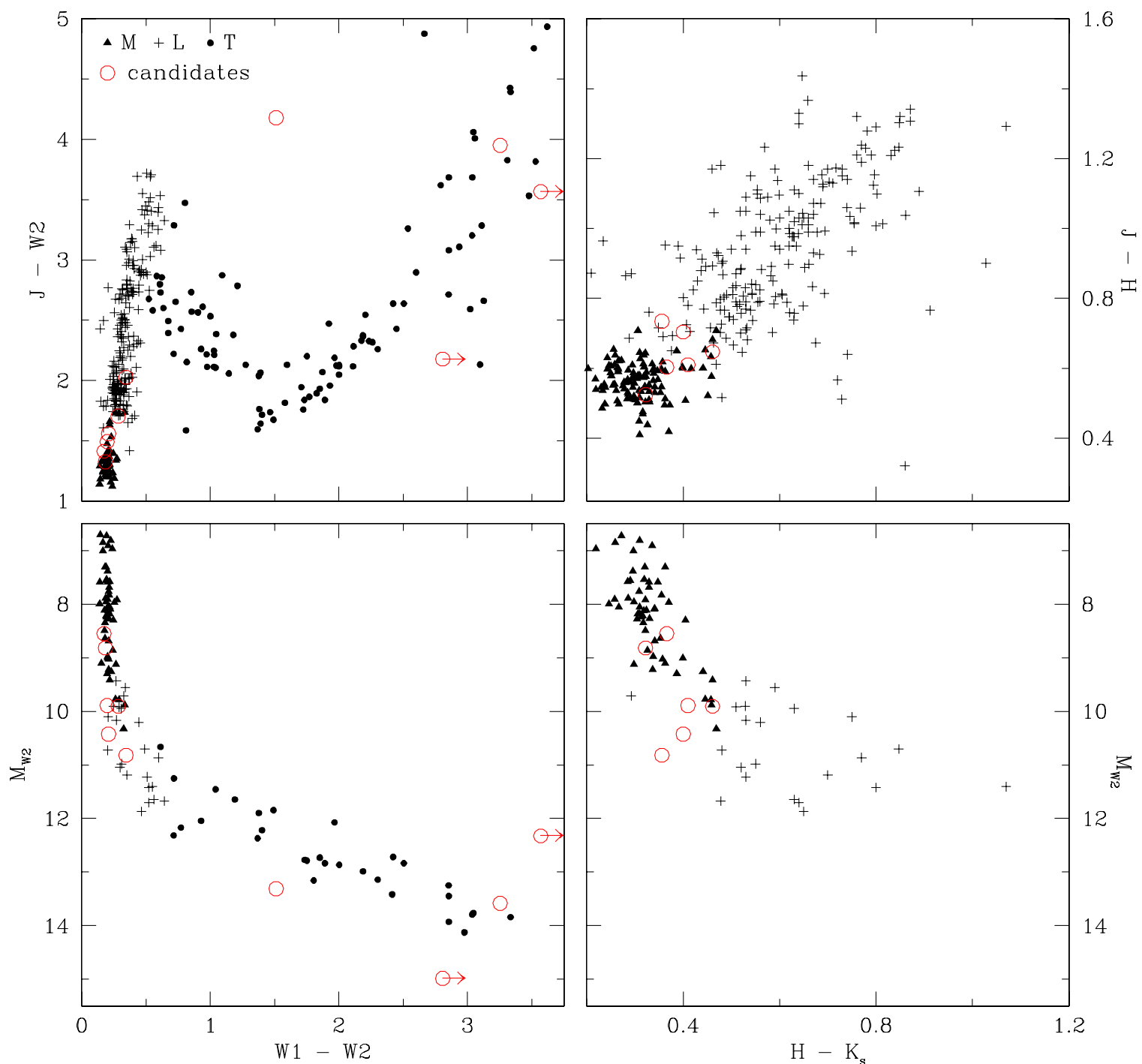

Figure 1. Color-magnitude and color-color diagrams for candidate late-type companions (open circles) and a sample of M, L, and T dwarfs (filled triangles, crosses, and filled circles; Leggett et al. 2010; Kirkpatrick et al. 2011, 2012; http://DwarfArchives.org). The T dwarfs are not shown in the diagrams on the right since our faintest candidates lack measurements of $K_{s}$. The candidate companions are assumed to have the same distances as their primaries for the bottom diagrams.

(A color version of this figure is available in the online journal.)

Table 2

Photometry for Candidate Companions

\begin{tabular}{|c|c|c|c|c|c|c|c|c|}
\hline Name & $J$ & $H$ & $K_{s}$ & Ref & $W 1$ & $W 2$ & $W 3$ & $W 4$ \\
\hline 2MASS $03184214+0828002$ & $13.79 \pm 0.03$ & $13.09 \pm 0.03$ & $12.69 \pm 0.02$ & 1 & $12.44 \pm 0.02$ & $12.23 \pm 0.03$ & $11.42 \pm 0.19$ & $\ldots$ \\
\hline WISE J041328.73+821854.7 & $\sim 19.2$ & $\ldots$ & $\ldots$ & 2 & $16.53 \pm 0.10$ & $15.02 \pm 0.09$ & $12.43 \pm 0.32$ & $\ldots$ \\
\hline ULAS J095047.28+011734.3 & $18.05 \pm 0.04$ & $18.24 \pm 0.15$ & $\ldots$ & 3 & $<18^{\mathrm{a}}$ & $14.48 \pm 0.06$ & & $\ldots$ \\
\hline 2MASS J10313234-5338010 & $11.84 \pm 0.02$ & $11.31 \pm 0.02$ & $10.99 \pm 0.02$ & 1 & $10.70 \pm 0.02$ & $10.51 \pm 0.02$ & $10.88 \pm 0.19$ & $\ldots$ \\
\hline WISE J142320.84+011638.0 & $18.71 \pm 0.05$ & $19.14 \pm 0.20$ & $\ldots$ & 4 & $18.01 \pm 0.30$ & $14.85 \pm 0.07$ & $\ldots$ & $\ldots$ \\
\hline 2MASS J14351087-2333025 & $13.54 \pm 0.02$ & $12.93 \pm 0.03$ & $12.52 \pm 0.03$ & 1 & $12.25 \pm 0.02$ & $12.05 \pm 02$ & $\ldots$ & $\ldots$ \\
\hline 2MASS J16220644+0101156 & $8.68 \pm 0.03$ & $8.00 \pm 0.02$ & $7.82 \pm 0.02$ & 1 & $7.69 \pm 0.02$ & $7.62 \pm 0.02$ & $7.54 \pm 0.02$ & $7.39 \pm 0.11$ \\
\hline 2MASS J17430860+8526594 & $14.56 \pm 0.04$ & $13.82 \pm 0.04$ & $13.47 \pm 0.05$ & 1 & $12.88 \pm 0.03$ & $12.53 \pm 0.03$ & $<12.4^{\mathrm{a}}$ & $\cdots$ \\
\hline WISE J180901.07+383805.4 & $17.37 \pm 0.05$ & & & 2 & $<18$ & $15.19 \pm 0.09$ & $\cdots$ & $\ldots$ \\
\hline 2MASS J18525777-5708141 & $12.03 \pm 0.02$ & $11.43 \pm 0.02$ & $11.06 \pm 0.02$ & 1 & $10.79 \pm 0.02$ & $10.62 \pm 0.02$ & $10.40 \pm 0.06$ & $\ldots$ \\
\hline 2MASS J20103539+0634367 & $12.53 \pm 0.02$ & $11.89 \pm 0.02$ & $11.43 \pm 0.02$ & 1 & $11.11 \pm 0.03$ & $10.83 \pm 0.02$ & $10.40 \pm 0.06$ & $\ldots$ \\
\hline
\end{tabular}

Notes. W1-W4 are from the WISE All-Sky Source Catalog.

a A detection is present in the WISE All-Sky Source Catalog, but none is apparent from visual inspection of the image.

References. (1) 2MASS Point Source Catalog; (2) this work; (3) UKIDSS Data Release 8; (4) Pinfield et al. 2012. 


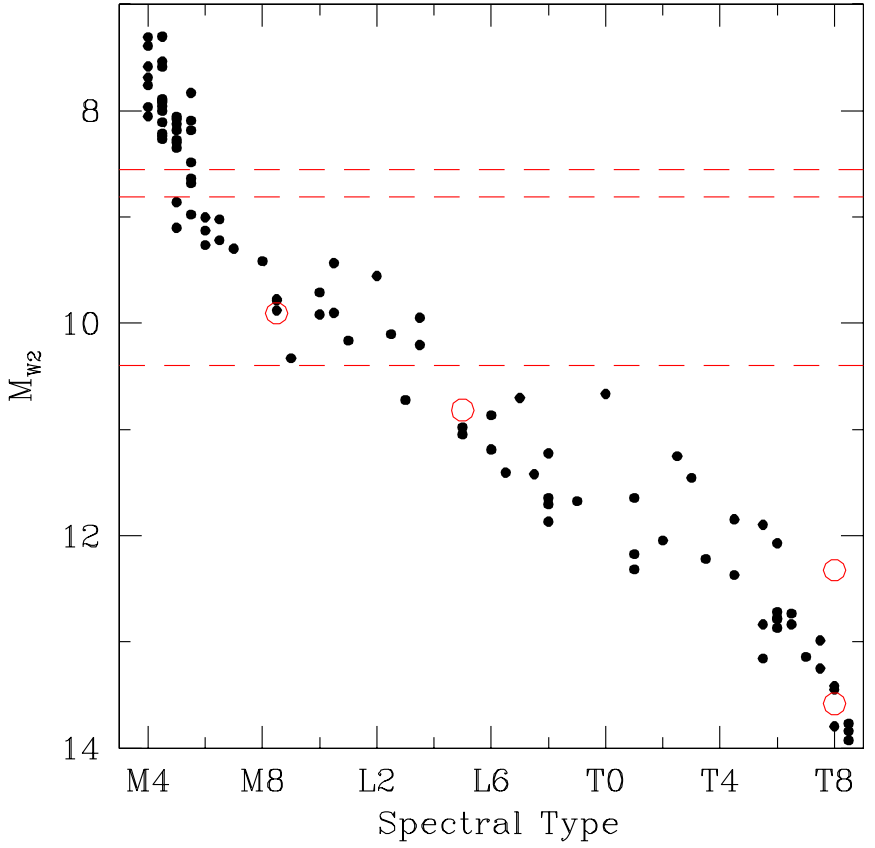

Figure 2. $M_{W 2}$ vs. spectral type for the candidate companions that exhibit common proper motions with their primaries (open circles and dashed lines; Table 1) and a sample of M, L, and T dwarfs with measured distances (filled circles; Leggett et al. 2010; Kirkpatrick et al. 2011, 2012; http://DwarfArchives.org).

(A color version of this figure is available in the online journal.)

of M3. LSPM J0318+0827N is unclassified, but it is probably later than the primary by $\sim 1$ subclass based on the relative fluxes of the pair $\left(\Delta K_{s}=1.0 \mathrm{mag}\right)$. We estimated a distance of 23 pc for LSPM J0318+0827S by combining its 2MASS photometry with the typical absolute magnitudes for an M3 dwarf (Kraus \& Hillenbrand 2007). At this distance, the $75^{\prime \prime}$ angular separation between 2M 0318+0828 and LSPM J0318+0827S corresponds to $1700 \mathrm{AU}$. The proper motion of 2M 0318+0828 measured from 2MASS and WISE agrees with that of LSPM J0318+0827S/N (Table 1), i.e., their $1 \sigma$ errors overlap. The colors and magnitudes of $2 \mathrm{M} 0318+0828$ are consistent with those expected for a late-M or early-L dwarf at the distance of the primary, as shown in Figures 1 and 2. Spectroscopy is needed to confirm that it has a spectral type in this range.

\subsection{WISE J041328.73+821854.7}

Our examination of WISE sources lacking 2MASS counterparts uncovered WISE J041328.73+821854.7 (hereafter WISE 0413+8218) as a candidate companion to LHS 1643. Law et al. (2008) estimated a spectral type of M4.5 for the latter from a measurement of $V-K$. The 2MASS photometry of LHS 1643 would then imply a distance of $22 \mathrm{pc}$. If this distance is adopted for WISE $0413+8218$, its position in $M_{W 2}$ versus $W 1-W 2$ is consistent with that of a T dwarf, as illustrated in Figure $1(W 1-W 2=1.5)$, and its angular separation of $215^{\prime \prime}$ would correspond to 4700 AU. Because this separation is small enough that companionship is plausible $(\lesssim 10,000 \mathrm{AU}$; Lépine \& Bongiorno 2007), we pursued follow-up imaging of WISE $0413+8218$ to measure its proper motion and better constrain its spectral type. Using the WIYN High-Resolution Infrared Camera (WHIRC), we obtained nine dithered $30 \mathrm{~s}$ exposures of WISE $0413+8218$ through a $J$-band filter on 2011 October 11. Only a weak detection of WISE $0413+8218$ appeared in the reduced mosaic of these data, but it was sufficient to demonstrate that the candidate is much redder in $J-W 2$ than expected for a $\mathrm{T}$ dwarf with its value of $W 1-W 2$ (Figure 1). In addition, no motion was detected for WISE $0413+8218$ between the WISE and WHIRC images, which is marginally inconsistent with companionship (Table 1). Therefore, we conclude that WISE $0413+8218$ is probably not a companion to LHS 1643 .

\subsection{ULAS J095047.28+011734.3}

The primary for our next candidate companion is LHS 6176. Because this star has not been previously classified, we performed spectroscopy on it with SpeX (Rayner et al. 2003) at the NASA Infrared Telescope Facility (IRTF). On the night of 2011 April 23, we observed LHS 6176 in the SXD mode with a 0 .'8 slit, which provided a wavelength coverage of $0.8-2.5 \mu \mathrm{m}$ and a resolution of $R=750$. The data were reduced with the Spextool package (Cushing et al. 2004) and corrected for telluric absorption (Vacca et al. 2003). The reduced spectrum is shown in Figure 3, where it has been smoothed to the resolution of the SpeX prism data that we have collected for some of our other candidates $(R=150)$. By comparing its spectrum to SpeX data for standard dwarfs (Cushing et al. 2005; Rayner et al. 2009), we measured a spectral type of M3.5V \pm 1 for LHS 6176. We estimated a distance of $27 \mathrm{pc}$ based on this classification and the photometry from 2MASS. Proper motions have been previously measured for LHS 6176 using DSS images (Bakos et al. 2002; Lépine \& Shara 2005). However, it is blended with another star in the images from the Second Palomar Sky Survey, which may have introduced errors into those data. Therefore, we have performed a new measurement using the first-epoch Palomar Sky Survey and archival Spitzer $4.5 \mu \mathrm{m}$ images from 2011 (Table 1).

The candidate companion to LHS 6176 is ULAS J095047.28+011734.3 (hereafter ULAS 0950+0117). B. Burningham (2012, in preparation) discovered this object using UKIDSS data and classified it as a T8 dwarf. We identified it as a possible companion through two separate surveys. In the first one, we searched for common proper motion companions using multi-epoch images from Spitzer (Luhman et al. 2011). Because ULAS 0950+0117 was observed by Spitzer in 2010 (program 60093 , S. Leggett), we selected it for a second epoch of imaging with the satellite in 2011 (program 70021, K. Luhman). By comparing the astrometry for all objects detected in both epochs, we found the proper motion of LHS 6176 was similar to that of ULAS 0950+0117. We later recovered ULAS 0950+0117 as a candidate companion during our analysis of WISE sources lacking 2MASS counterparts that are in close proximity to nearby stars like LHS 6176 (Section 2).

ULAS 0950+0117 and LHS 6176 have an angular separation of 52", corresponding to $1400 \mathrm{AU}$ at the distance of the latter. UKIDSS provides the earliest astrometry of ULAS 0950+0117. It was detected in $H$ and $K$ in 2006 and 2007, but the signalto-noise ratios were low. Therefore, we use the $J$-band data from 2008 February 23 for the first epoch in our measurement of the proper motion of ULAS 0950+0117. For the second epoch, we used the most recent publicly available images that were taken near the same time of year as the first epoch to minimize parallactic motion, which consist of $J$-band data acquired on the night of 2012 March 3 with SOFI on the European Southern Observatory New Technology Telescope (NTT, program 186.C-0756, R. Smart). The resulting proper motion measurement for ULAS $0950+0117$ is similar to that of LHS 6176 such that their $1 \sigma$ error bars overlap (Table 1). The diagram of $M_{W 2}$ versus spectral type in Figure 2 shows that ULAS 0950+0117 is brighter than expected for a T8 dwarf 


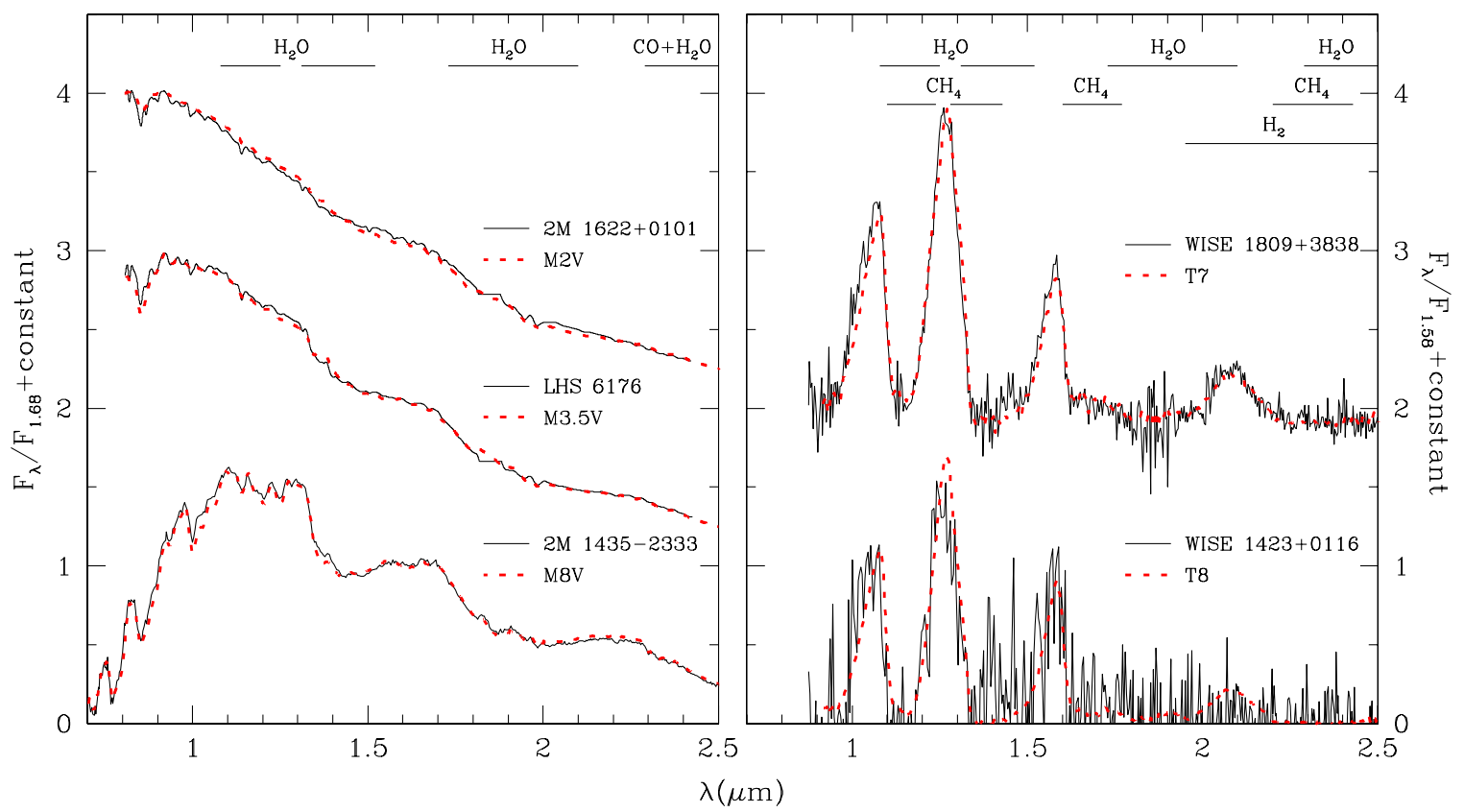

Figure 3. Near-IR spectra of the primary star LHS 6176 and four of the candidate companions (solid lines) compared to data for dwarf standards (dotted lines; Cushing et al. 2005; Rayner et al. 2009; Burgasser et al. 2004, 2006). The data are displayed at a resolution of $R=150$.

(A color version of this figure is available in the online journal.)

at the spectrophotometric distance of LHS 6176, which may indicate that it is an unresolved binary. ULAS $0950+0117$ has been imaged several times over the last few years with both the NTT and the Canada-France-Hawaii Telescope (CFHT) in an effort to measure its parallax (Marocco et al. 2010; Dupuy \& Liu 2012), which will eventually provide an additional test of its companionship and overluminous nature.

\subsection{MASS J10313234-5338010}

2MASS J10313234-5338010 (hereafter 2M 1031-5338) was found as a possible companion to HD 91324 (F9V, $21.8 \mathrm{pc}$ ) through our 2MASS/WISE proper motion survey. The angular separation of the two stars is $309^{\prime \prime}$ (6700 AU). Our proper motion measurement for 2M 1031-5338 from 2MASS and WISE is within $1 \sigma$ of that of HD 91324 (Table 1). Using Figures 1 and 2, we find that the photometry of 2M 1031-5338 is indicative of an M5-M6 dwarf near the distance of HD 91324. As with 2M 0318+0828, spectroscopy is needed to verify this spectral type.

\subsection{WISE J142320.84+011638.0}

We identified WISE J142320.84+011638.0 (hereafter WISE 1423+0116) as a candidate companion to LHS 2907 (BD+01 2920$)$ from among WISE sources lacking 2MASS counterparts. LHS 2907 is a moderately metal-poor solar-type star $([\mathrm{Fe} / \mathrm{H}] \sim-0.35$; Valenti \& Fischer 2005; Casagrande et al. 2011). The pair has an angular separation of $156^{\prime \prime}$ or 2680 AU at the distance of the primary $(17.2 \mathrm{pc})$. After checking data archives from various surveys and observatories, we found detections of WISE $1423+0116$ in $Y$ - and $J$-band images from UKIDSS and $J$-band images from WIRCam at the CFHT (program 09BD95, L. Albert). The values of $W 2, W 1-W 2, J-W 2$ are consistent with a late T dwarf at the distance of LHS 2907 (Figure 1). Using the images from UKIDSS (2008 May 16) and CFHT (2009 December 29), we measured the proper motion of WISE 1423+0116, which was found to be similar to that of LHS 2907 (Table 1). Because of this promising evidence that WISE 1423+0116 was a T dwarf companion, we obtained a spectrum of it with SpeX at the IRTF. These data were collected in the prism mode with a $0{ }^{\prime} .8$ slit, providing a wavelength coverage of $0.8-2.5 \mu \mathrm{m}$ and a resolution of $R=150$. The spectrum exhibits the strong absorption bands that characterize late $\mathrm{T}$ dwarfs, as illustrated in Figure 3 where we compare it to data for a standard T8 dwarf (2MASS J04151954-0935066; Burgasser et al. 2004). During their independent discovery of WISE 1423+0116, Pinfield et al. (2012) obtained a spectrum with higher signal-to-noise ratio, producing a more accurate classification than is possible with our data. Using their spectral type of T8 and the distance of the primary, WISE $1423+0116$ falls within the sequence of T dwarfs in the diagram of $M_{W 2}$ versus spectral type in Figure 2, which supports its companionship.

\subsection{MASS J14351087-2333025}

Our 2MASS/WISE proper motion search uncovered 2MASS J14351087-2333025 (hereafter 2M 1435-2333) as a possible companion to 2MASS J14345819-2335572 (hereafter 2M 1434-2335). The latter has a spectral type of M7V and a corresponding distance of $27 \mathrm{pc}$. The pair had an angular separation of $247^{\prime \prime}$ in the 2MASS images (6700 AU). The proper motion that we measure for 2M 1435-2333 using the first-epoch Palomar Sky Survey, 2MASS, and WISE (Table 1) agrees with the value of $\left(\mu_{\alpha}, \mu_{\delta}\right)=(-306 \pm 30,+51 \pm 5)$ mas $\mathrm{yr}^{-1}$ for $2 \mathrm{M} 1434-2335$ from Faherty et al. (2009). To further characterize 2M 1435-2333, we obtained a spectrum of it using the prism mode of SpeX on the night of 2012 May 6. The spectrum matches our SpeX data for the M8V standard 2MASS J03205965+1854233 (Kirkpatrick et al. 1995), as shown in Figure 3. Our classification of $\mathrm{M} 8 \mathrm{~V} \pm 0.5$ is roughly consistent with companionship given its photometry. However, when we measure the proper motion of $2 \mathrm{M}$ 1434-2335 


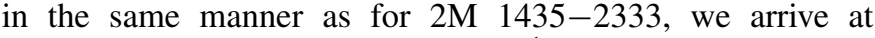
$\left(\mu_{\alpha}, \mu_{\delta}\right)=(-314,-19) \pm 15 \mathrm{mas} \mathrm{yr}^{-1}$, which differs from the motion from Faherty et al. (2009). Thus, the motions that we measure for the components of this pair indicate that they are not companions.

\subsection{MASS J16220644+0101156}

2MASS J16220644+0101156 (hereafter 2M 1622+0101) is a candidate companion to HD 147449 (F0V, 27.3 pc) based on our 2MASS/WISE proper motion analysis. 2M 1622+0101 is relatively close to HD 147449 with an angular separation of only 43" (1200 AU). The proper motion of 2M 1622+0101 that we measured from 2MASS and WISE agrees with that of HD 147449 (Table 1). To measure the spectral type of 2M 1622+0101, we performed spectroscopy on it with SpeX in its SXD mode $(R=750)$ on the night of 2012 April 24. Through comparison to SpeX data for standard stars (Cushing et al. 2005; Rayner et al. 2009), we classified 2M 1622+0101 as $\mathrm{M} 2 \mathrm{~V} \pm 1$. The spectrum of $2 \mathrm{M} 1622+0101$ is plotted with data for the M2V star G1 411 in Figure 3, both of which have been smoothed to the resolution of the prism spectra $(R=150)$. Our classification is consistent with the expected spectral type based on its photometry if it has the same distance as HD 147449. Because of its relatively early spectral type, 2M 1622+0101 does not appear within the boundaries of Figures 1 and 2.

\subsection{MASS J17430860+8526594}

Using 2MASS and WISE, we identified 2MASS J17430860+ 8526594 (hereafter 2M 1743+8526) as a candidate companion to G259-20. Neither a spectral type nor a parallax has been measured previously for G259-20, but its optical and IR photometry are indicative of an early-M dwarf at 20-30 pc. The proper motion of $2 \mathrm{M} 1743+8526$ based on 2MASS and WISE agrees with that of G259-20 (Table 1) and the pair has a relatively small separation $\left(30^{\prime \prime}, 650 \mathrm{AU}\right)$. In addition, the photometry of $2 \mathrm{M} 1743+8526$ is consistent with that of a late-type object near the distance of G259-20 (Figure 1). Because of the promising nature of this candidate, we pursued spectroscopy of it and G259-20 with the near-infrared spectrometer (NIRSPEC; McLean et al. 1998, 2000) on the 10 m telescope at W. M. Keck Observatory on the night of 2012 June 7. The instrument was operated in its low-resolution mode with the N3 filter and 0.'57 slit, which provides spectra across the $J$ band with a resolution of $R=1700$. The data were reduced with the REDSPEC package (McLean et al. 2003) and the resulting spectra are presented in Figure 4. We measured spectral types of $\mathrm{M} 2.5 \mathrm{~V} \pm 1$ and L5 \pm 1 for G259-20 and 2M 1743+8526, respectively, using spectra of standards obtained with SpeX (Cushing et al. 2005; Rayner et al. 2009) and NIRSPEC (McLean et al. 2003). Based on the metallicities of the early-M SpeX standards (e.g., Rojas-Ayala et al. 2012), we estimate that G259-20 has $[\mathrm{Fe} / \mathrm{H}] \approx-0.2$ to 0 , which is relevant to the interpretation of unusual colors for $2 \mathrm{M} \mathrm{1743+8526}$ (Section 4). In Figure 4, we show the standards that produced the best matches, consisting of G1 581 (M2.5V) for the primary and DENIS-P J1228.2-1547 (L5) for the secondary. When combined with its 2MASS photometry, a spectral type of M2.5V for G259-20 implies a distance of 22 pc. $2 \mathrm{M} 1743+8526$ is plotted on the diagram of $M_{W 2}$ versus spectral type in Figure 2 assuming this distance, which places it within the sequence of known L dwarfs, as expected for a companion.

\subsection{WISE J180901.07+383805.4}

We identified WISE J180901.07+383805.4 (hereafter WISE $1809+3838)$ as a candidate companion to HD 166620 $(\mathrm{K} 2 \mathrm{~V}, 11.0 \mathrm{pc})$ from among WISE sources that do not appear in 2MASS. The angular separation is 769", or $8460 \mathrm{AU}$ at the distance of HD 166620. Given its WISE photometry $(W 1-W 2>2.8)$, WISE $1809+3838$ could be a companion to HD 166620 with a spectral type of $>\mathrm{T} 9$, as shown in Figure 1. We found a detection of WISE $1809+3838$ in archival $J$-band images obtained by WIRCam at the CFHT (program 08BD95, L. Albert) on the night of 2008 October 12. The photometry that we measured from these data indicates a color of $J-W 2=2.2$, which is bluer than expected for a > T9 companion. Nevertheless, to investigate the nature of WISE 1809+3838 further, we observed it with SpeX in the prism mode on the night of 2011 June 28 . The resulting spectrum is presented in Figure 3, where we find a good match to the T7 standard 2MASS J07271824+1710012 (Burgasser et al. 2006). The uncertainty in this classification is \pm 1 subclass. If WISE $1809+3838$ has an absolute magnitude in $W 2$ that is similar to that of typical T7 dwarfs, then it should have a distance of roughly $28 \mathrm{pc}$, indicating that it is probably not a companion to HD 166620. In addition to the spectroscopic data, we obtained $J$-band images of WISE $1809+3838$ with the slit-viewing camera on SpeX. The proper motion of WISE 1809+3838 measured from the SpeX and WIRCam data is inconsistent with that of HD 166620 (Table 1), confirming that the pair is not a binary system.

\subsection{MASS J18525777-5708141}

2MASS J18525777-5708141 (hereafter 2M 1852-5708) was found as a candidate companion to LHS 3421 (M2.5V, $25.9 \mathrm{pc}$ ) through our 2MASS/WISE proper motion search. The pair has an angular separation of 58" (1500 AU). The proper motion of 2M 1031-5338 based on 2MASS and WISE is within $1 \sigma$ of that of LHS 3421 (Table 1). The colors and magnitudes of 2M 1852-5708 are consistent with those of a dwarf between M5 and M6 at the distance of the primary (Figures 1 and 2).

\subsection{MASS J20103539+0634367}

We uncovered 2MASS J20103539+0634367 (hereafter 2M 2010+0634) as a candidate companion to the M-type spectroscopic binary LSPM J2010+0632 with data from 2MASS and WISE. The latter has a distance of $15.3 \mathrm{pc}$ based on its spectral type and photometry (Shkolnik et al. 2010). The angular separation of the pair is $143^{\prime \prime}$ (2100 AU). The proper motion of 2M 2010+0634 derived from 2MASS and WISE agrees with that of LSPM J2010+0632 (Table 1). The photometry of 2M 2010+0634 is also consistent with a spectral type of late $\mathrm{M}$ or early $\mathrm{L}$ at the distance of the primary (Figure 1). To verify its spectral type, we obtained a spectrum of 2M 2010+0634 with the Marcario Low-Resolution Spectrograph (LRS) on the Hobby-Eberly Telescope (HET) on the night of 2011 August 26 . The instrument was operated with the G3 grism and the $2^{\prime \prime}$ slit, which provided a wavelength coverage of 6200-9100 $\AA$ and a resolution of $R=1100$. As illustrated in Figure 5, the spectrum agrees well with a combined spectrum of VB 10 (M8V), LHS 2243 (M8V), and LHS 2243 (M9V) in which the latter was given twice the weighting of each of the other two stars. Therefore, we classified $2 \mathrm{M} 2010+0634$ as $\mathrm{M} 8.5 \mathrm{~V} \pm 0.5$. Using this spectral type and the distance of the primary, 2M 2010+0634 


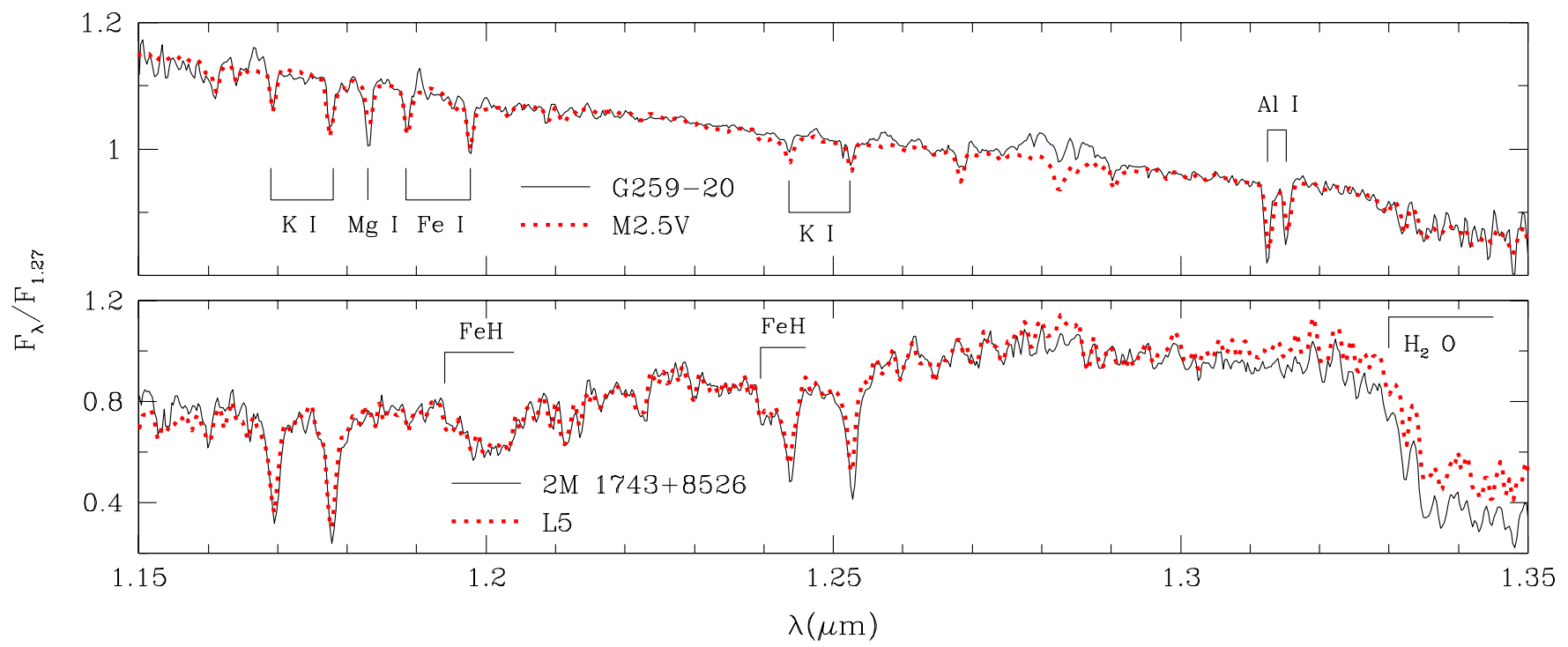

Figure 4. Near-IR spectra of the primary star G259-20 and its candidate companion 2M 1743+8526 (solid lines) compared to data for dwarf standards (dotted lines). (A color version of this figure is available in the online journal.)

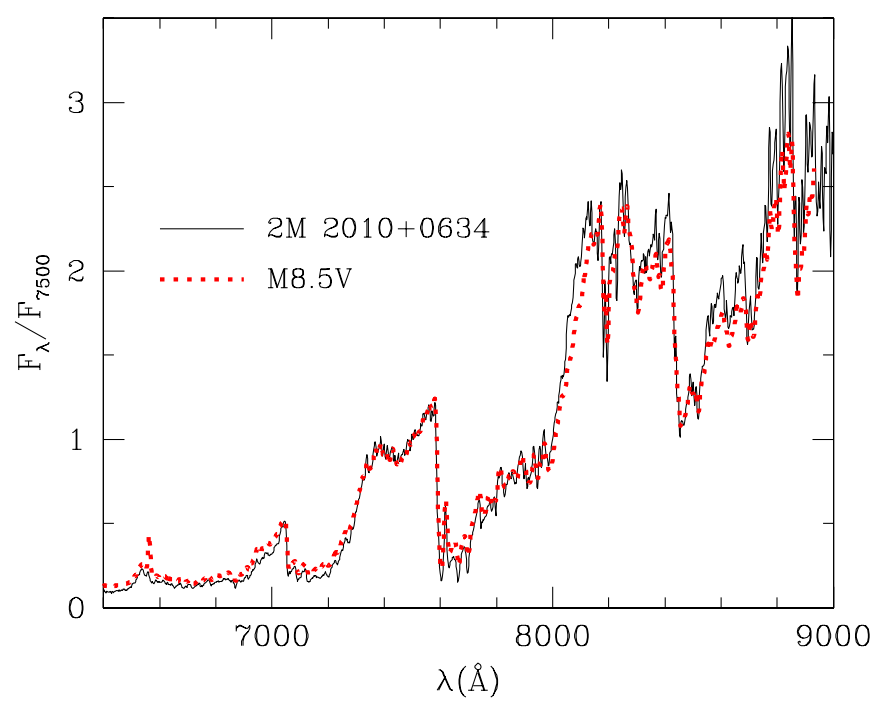

Figure 5. Optical spectrum of the candidate companion 2M 2010+0634 (solid line) compared to the average of data for M8V and M9V standards (dotted line). (A color version of this figure is available in the online journal.)

appears within the sequence of $\mathrm{M}$ dwarfs in Figure 2, which supports its companionship.

\section{DISCUSSION}

We have presented 11 candidate late-type companions to nearby stars that were found with two methods involving data from WISE and 2MASS. In general, the probability of companionship for a candidate depends on the size of its proper motion $(\mu)$, the differences between the proper motions and distances of the candidate and the primary $(\Delta \mu, \Delta d)$, the uncertainties in those motions and distances $\left(\sigma_{\mu}, \sigma_{d}\right)$, and the angular separation of the pair $(\Delta \theta)$. For eight of our candidates, the values of these parameters are comparable to those of cool dwarfs that have been previously accepted as companions. For instance, these objects satisfy by wide margins the criteria for companionship proposed by Lépine \& Bongiorno (2007; $\left.\Delta \theta \Delta \mu<(\mu / 0.15)^{3.8}\right)$ and Dupuy \& Liu $(2012 ; \Delta \mu / \mu<0.2)$.
These likely companions, which are indicated in Table 1 , include two $\mathrm{M}$ dwarfs, one $\mathrm{L}$ dwarf, and two $\mathrm{T}$ dwarfs. The three remaining companions lack spectroscopic classifications, but they probably have $\mathrm{M} / \mathrm{L}$ types based on their photometry.

Cooler companions have greater value for testing model atmospheres and interiors. The T dwarf companion WISE 1423+0116 was independently discovered and previously reported by Pinfield et al. (2012), who thoroughly examined its physical properties. Our remaining L/T companions are ULAS $0950+0117$ and $2 \mathrm{M} 1743+8526$. Because their primaries are not well studied (e.g., lack parallax data), it is not currently possible to accurately estimate their properties and fully exploit them for calibrating models. However, both companions exhibit distinctive characteristics that warrant discussion. If it is a companion, ULAS $0950+0117$ is brighter than typical $\mathrm{T}$ dwarfs at its spectral type according to the primary's spectrophotometric distance, indicating that it may be an unresolved binary. Thus, estimating the properties of this object for comparison to models will require better constraints on its binarity via high-resolution imaging. If confirmed as a binary, ULAS 0950+0117 would join a small group of known $\mathrm{L}$ and $\mathrm{T}$ dwarf binaries in orbit around stars (Wilson et al. 2001; Potter et al. 2002; Bouy et al. 2003; McCaughrean et al. 2004; Burgasser et al. 2005). Such pairs are especially useful for testing theoretical models since their ages and metallicities can be estimated via the stellar primaries while their masses can be measured dynamically (Dupuy et al. 2009; Konopacky et al. 2010).

$2 \mathrm{M} 1743+8526$ is also notable in that it has unusually blue near-IR colors for its spectral type $\left(J-K_{s}=1.09 \pm 0.06\right)$. A number of blue $\mathrm{L}$ dwarfs have been previously identified (Cruz et al. 2003; Knapp et al. 2004; Kirkpatrick et al. 2010, references therein). Two of the most prominent examples, 2MASS J11263991-5003550 (Burgasser et al. 2008a) and SDSS J141624.08+134826.7 (hereafter SDSS 1416+1348; Bowler et al. 2010; Schmidt et al. 2010), resemble $2 \mathrm{M} 1743+8526$ in terms of colors and spectral type. The $1.15-1.3 \mu \mathrm{m}$ spectral features of $2 \mathrm{M} 1743+8526$ match those of normal L5 dwarfs, but it has stronger $\mathrm{H}_{2} \mathrm{O}$ absorption at $1.33-1.35 \mu \mathrm{m}$ (Figure 4), which is similar to the behavior of SDSS 1416+1348 (Schmidt et al. 2010). A comparison between 
2M 1743+8526 and these two L dwarfs at other wavelengths would be useful for determining whether they share other spectral characteristics as well, although observations of the former are hampered by its declination, which places it at high airmass and above the declination limits of some telescopes.

Possible explanations for the peculiar colors of blue L dwarfs include low metallicity, unresolved companions, and condensate clouds that are thin or have large grains. Most studies have favored the latter (Burgasser et al. 2008a; Bowler et al. 2010; Cushing et al. 2010), but the properties of the T dwarf companion to SDSS $1416+1348$ suggest that low metallicity plays a role as well (Dupuy \& Liu 2012). Because some of its properties can be estimated through its primary star, 2M 1743+8526 offers a new opportunity for constraining these proposed mechanisms. Adopting the spectrophotometric distance of its primary, the absolute magnitudes of $2 \mathrm{M} 1743+8526$ are brighter than the average values for L5 dwarfs (Dupuy \& Liu 2012) by 0.72 $(J), 0.38(H), 0.17\left(K_{s}\right), 0.01(W 1)$, and $0.03 \mathrm{mag}(W 2)$. The enhanced $J$-band flux is consistent with the third scenario described above (Burgasser et al. 2008b). Meanwhile, our metallicity estimate for the primary $([\mathrm{Fe} / \mathrm{H}] \approx-0.2$ to 0$)$ indicates that it is not an outlier in the metallicity distribution in the solar neighborhood (Nordström et al. 2004), and hence low metallicity is probably not the cause of $2 \mathrm{M} \mathrm{1743+8526}$ 's unusual colors. Radigan et al. (2008) arrived at a similar result through analysis of the primary for the blue L5 companion 2MASS J17114559+4028578 $\left(J-K_{s}=1.21 \pm 0.08\right)$. Thus, the data for $2 \mathrm{M} 1743+8526$ support the previous suggestion that thin condensate clouds can account for some blue L dwarfs.

K.L. acknowledges support from grant AST-0544588 from the National Science Foundation (NSF) and grant NNX12AI47G from the NASA Astrophysics Data Analysis Program. WISE is a joint project of the University of California, Los Angeles, and the Jet Propulsion Laboratory (JPL)/California Institute of Technology (Caltech), funded by NASA. 2MASS is a joint project of the University of Massachusetts and the Infrared Processing and Analysis Center (IPAC) at Caltech, funded by NASA and the NSF. The W. M. Keck Observatory is operated as a scientific partnership among Caltech, the University of California, and NASA and was made possible by the generous financial support of the W. M. Keck Foundation. The IRTF is operated by the University of Hawaii under cooperative agreement NNX-08AE38A with NASA. The HET is a joint project of the University of Texas at Austin, the Pennsylvania State University, Stanford University, Ludwig-MaximilliansUniversität München, and Georg-August-Universität Göttingen and is named in honor of its principal benefactors, William P. Hobby and Robert E. Eberly. The Spitzer Space Telescope is operated by JPL and Caltech under contract with NASA. Kitt Peak National Observatory is operated by the Association of Universities for Research in Astronomy, Inc. under cooperative agreement with the NSF. WIYN Observatory is a joint facility of the University of Wisconsin-Madison, Indiana University, Yale University, and the National Optical Astronomy Observatory. WIRCam is a joint project of CFHT, Taiwan, Korea, Canada, and France. CFHT is operated by the National Research Council of Canada, the Institute National des Sciences de l'Univers of the Centre National de la Recherche Scientifique of France, and the University of Hawaii. This work uses data from the SpeX Prism Spectral Libraries (maintained by Adam Burgasser at http://www.browndwarfs.org/spexprism), the M, L, and T dwarf compendium at http://DwarfArchives.org (maintained by Chris Gelino, Davy Kirkpatrick, and Adam Burgasser), the Brown Dwarf Spectroscopic Survey Archive (http://www.astro.ucla.edu/ mclean/BDSSarchive), the NASA/IPAC Infrared Science Archive (operated by JPL under contract with NASA), and the ESO/ST-ECF Science Archive Facility. The Center for Exoplanets and Habitable Worlds is supported by the Pennsylvania State University, the Eberly College of Science, and the Pennsylvania Space Grant Consortium. The authors wish to recognize and acknowledge the very significant cultural role and reverence that the summit of Mauna Kea has always had within the indigenous Hawaiian community. We are most fortunate to have the opportunity to conduct observations from this mountain.

\section{REFERENCES}

Allard, F., Homeier, D., \& Freytag, B. 2011, Phil. Trans. R. Soc. A, 370, 2765 Bakos, G. A., Sahu, K. C., \& Nemeth, P. 2002, ApJS, 141, 187

Baraffe, I., Chabrier, G., Barman, T. S., Allard, F., \& Hauschildt, P. H. 2003, A\&A, 402, 701

Becklin, E. E., \& Zuckerman, B. 1988, Nature, 336, 656

Biller, B. A., Kasper, M., Close, L. M., Brandner, W., \& Kellner, S. 2006, ApJ, 641, L141

Bouy, H., Brandner, W., Martín, E. L., et al. 2003, AJ, 126, 1526

Bowler, B. P., Liu, M. C., \& Dupuy, T. J. 2010, ApJ, 710, 45

Burgasser, A. J., Burrows, A., \& Kirkpatrick, J. D. 2006, ApJ, 639, 1095

Burgasser, A. J., Kirkpatrick, J. D., Cutri, R. M., et al. 2000, ApJ, 531, L57

Burgasser, A. J., Kirkpatrick, J. D., \& Lowrance, P. J. 2005, AJ, 129, 2849

Burgasser, A. J., Looper, D. L., Kirkpatrick, J. D., Cruz, K. L., \& Swift, B. J 2008a, ApJ, 674, 451

Burgasser, A. J., McElwain, M. W., \& Kirkpatrick, J. D. 2004, AJ, 127, 2856

Burgasser, A. J., Vrba, F. J., Lépine, S., et al. 2008b, ApJ, 672, 1159

Burningham, B., Pinfield, D. J., Leggett, S. K., et al. 2009, MNRAS, 395, 1237

Burrows, A., Heng, K., \& Nampaisarn, N. 2011, ApJ, 736, 47

Casagrande, L., Schönrich, R., Asplund, M., et al. 2011, A\&A, 530, 138

Cowley, A. P., Hiltner, W. A., \& Witt, A. N. 1967, AJ, 72, 1334

Cruz, K. L., Reid, I. N., Kirkpatrick, J. D., et al. 2007, AJ, 133, 439

Cruz, K. L., Reid, I. N., Liebert, J., Kirkpatrick, J. D., \& Lowrance, P. J. 2003, AJ, 126,2421

Cushing, M. C., Kirkpatrick, J. D., Gelino, C. R., et al. 2011, ApJ, 743, 50

Cushing, M. C., Rayner, J. T., \& Vacca, W. D. 2005, ApJ, 623, 1115

Cushing, M. C., Saumon, D., \& Marley, M. S. 2010, AJ, 140, 1428

Cushing, M. C., Vacca, W. D., \& Rayner, J. T. 2004, PASP, 116, 362

Day-Jones, A. C., Pinfield, D. J., Ruiz, M. T., et al. 2011, MNRAS, 410, 705

Deacon, N. R., Liu, M. C., Magnier, E. A., et al. 2011, AJ, 142, 77

Deacon, N. R., Liu, M. C., Magnier, E. A., et al. 2012a, ApJ, 755, 94

Deacon, N. R., Liu, M. C., Magnier, E. A., et al. 2012b, ApJ, 757, 100

Dupuy, T. J., \& Liu, M. C. 2012, ApJS, 201, 19

Dupuy, T. J., Liu, M. C., \& Ireland, M. J. 2009, ApJ, 692, 729

Faherty, J. K., Burgasser, A. J., Cruz, K. L., et al. 2009, AJ, 137, 1

Faherty, J. K., Burgasser, A. J., West, A. A., et al. 2010, AJ, 139, 176

Gizis, J. E., Kirkpatrick, J. D., \& Wilson, J. C. 2001, AJ, 121, 2185

Goldman, B., Marsat, S., Henning, T., Clemens, C., \& Greiner, J. 2010, MNRAS, 405, 1140

Gray, R. O., Corbally, C. J., Garrison, R. F., et al. 2006, AJ, 132, 161

Hambly, N. C., MacGillivray, H. T., Read, M. A., et al. 2001, MNRAS, 326,1279

Hawley, S. L., Gizis, J. E., \& Reid, I. N. 1996, AJ, 112, 2799

Høg, E., Fabricius, C., Makarov, V. V., et al. 2000, A\&A, 355, L27

Kaiser, N., Aussel, H., Burke, B. E., et al. 2002, Proc. SPIE, 4836, 154

Kirkpatrick, J. D., Cushing, M. C., Gelino, C. R., et al. 2011, ApJS, 197, 19

Kirkpatrick, J. D., Dahn, C. C., Monet, D. G., et al. 2001, AJ, 127, 3235

Kirkpatrick, J. D., Gelino, C. R., Cushing, M. C., et al. 2012, ApJ, 753, 156

Kirkpatrick, J. D., Henry, T. J., \& Simons, D. A. 1995, AJ, 109, 797

Kirkpatrick, J. D., Looper, D. L., Burgasser, A. J., et al. 2010, ApJS, 190, 100

Knapp, G. R., Leggett, S. K., Fan, X., et al. 2004, AJ, 127, 3553

Konopacky, Q. M., Ghez, A. M., Barman, T. S., et al. 2010, ApJ, 711, 1087

Kraus, A. L., \& Hillenbrand, L. A. 2007, AJ, 134, 2340

Law, N. M., Hodgkin, S. T., \& Mackay, C. D. 2008, MNRAS, 384, 150

Lawrence, A., Warren, S. J., Almaini, O., et al. 2007, MNRAS, 379, 1599

Leggett, S. K., Burningham, B., Saumon, D., et al. 2010, ApJ, 710, 1627

Lépine, S. 2005, AJ, 130, 1680

Lépine, S. 2008, AJ, 135, 2177

Lépine, S., \& Bongiorno, B. 2007, AJ, 133, 889 
Lépine, S., \& Shara, M. M. 2005, AJ, 129, 1483

Liu, M. C., Deacon, N. R., Magnier, E. A., et al. 2011, ApJ, 740, L32

Loutrel, N. P., Luhman, K. L., Lowrance, P. J., \& Bochanski, J. J. 2011, ApJ, 739,81

Luhman, K. L., Burgasser, A. J., \& Bochanski, J. J. 2011, ApJ, 730, L9

Luhman, K. L., Patten, B. M., Marengo, M., et al. 2007, ApJ, 654, 570

Marocco, F., Smart, R. L., Jones, H. R. A., et al. 2010, A\&A, 524, A38

McCaughrean, M. J., Close, L. M., Scholz, R.-D., et al. 2004, A\&A, 413, 1029

McLean, I. S., Becklin, E. E., Bendiksen, O., et al. 1998, Proc. SPIE, 3354, 566

McLean, I. S., Graham, J. R., Becklin, E. E., et al. 2000, Proc. SPIE, 4008, 1048

McLean, I. S., McGovern, M. R., Burgasser, A. J., et al. 2003, ApJ, 596, 561

Metchev, S., \& Hillenbrand, L. 2006, ApJ, 651, 1166

Mugrauer, M., Seifahrt, A., Neuhäuser, R., \& Mazeh, T. 2006, MNRAS, 373, L31

Mužić, K., Radigan, J., Jayawardhana, R., et al. 2012, AJ, in press

Nakajima, T., Oppenheimer, B. R., Kulkarni, S. R., et al. 1995, Nature, 378, 463

Nordström, B., Mayor, M., Andersen, J., et al. 2004, A\&A, 418, 989

Pinfield, D. J., Burningham, B., Lodieu, N., et al. 2012, MNRAS, 422, 1922

Potter, D., Martin, E. L., Cushing, M. C., et al. 2002, ApJ, 567, L133

Radigan, J., Lafreniére, D., Jayawardhana, R., \& Doyon, R. 2008, ApJ, 689,471

Rayner, J. T., Cushing, M. C., \& Vacca, W. D. 2009, ApJS, 185, 289

Rayner, J. T., Toomey, D. W., Onaka, P. M., et al. 2003, PASP, 115, 362

Robertson, T. H. 1984, AJ, 89, 1229
Rojas-Ayala, B., Covey, K. R., Muirhead, P. S., \& Lloyd, J. P. 2012, ApJ 748,93

Saumon, D., Marley, M. S., Abel, M., Frommhold, L., \& Freedman, R. S. 2012, ApJ, 750, 74

Schmidt, S. J., West, A. A., Burgasser, A. J., Bochanski, J. J., \& Hawley, S. L. 2010, AJ, 139, 1045

Scholz, R.-D. 2010, A\&A, 515, A92

Scholz, R.-D., McCaughrean, M. J., Lodieu, N., \& Kuhlbrodt, B. 2003, A\&A 398, L29

Shkolnik, E. L., Hebb, L., Liu, M. C., Reid, I. N., \& Cameron, A. C. 2010, ApJ, 716,1522

Skrutskie, M., Cutri, R. M., Stiening, R., et al. 2006, AJ, 131, 1163

Slettebak, A. 1955, ApJ, 121, 653

Subasavage, J. P., Henry, T. J., Hambly, N. C., et al. 2005, AJ, 130, 1658

Thalmann, C., Carson, J., Janson, M., et al. 2009, ApJ, 707, L123

Vacca, W. D., Cushing, M. C., \& Rayner, J. T. 2003, PASP, 115, 389

Valenti, J. A., \& Fischer, D. A. 2005, ApJS, 159, 141

van Leeuwen, F. 2007, A\&A, 474, 653

Werner, M. W., Roellig, T. L., Low, F. J., et al. 2004, ApJS, 154, 1

Wilson, J. C., Kirkpatrick, J. D., Gizis, J. E., et al. 2001, AJ, 122, 1989

Wright, E. L., Eisenhardt, P. R. M., Mainzer, A. K., et al. 2010, AJ, 140, 1868

York, D. G., Adelman, J., Anderson, J. E., et al. 2000, AJ, 120, 1579

Zhang, Z. H., Pinfield, D. J., Day-Jones, A. C., et al. 2010, MNRAS, 404, 1817 